\title{
Late-order terms of second order ODEs in terms of pre-factors
}

\author{
Fatih Say (1D \\ Department of Mathematics, Faculty of Arts and Sciences, Ordu University, Ordu, Turkey
}

\begin{abstract}
Factorial over a power approach is one of the fundamental techniques for deriving the late-order terms in the asymptotic approximation of integrals and differential equations. To our knowledge, although many differential equations depending on small or large parameters are addressed thoroughly and intensively by this approach in the literature to date, no explicit formula of the general representation of singularly-perturbed second order inhomogeneous ODEs in the form of this paper has yet been discussed generally in terms of their pre-factors. In this paper, we obtain a leading order asymptotic formula of the general asymptotic expansions suitable for the particular type of ODE by its pre-factors.
\end{abstract}

Mathematics Subject Classification (2020). 34E10, 34E15

Keywords. asymptotic approximation, asymptotic expansion, asymptotic beyond all orders, singular perturbation

\section{Introduction}

Factorial over a power (factorial/power) is one of the best-known methods for developing asymptotic approximations of differential equations and integrals. The origins of the method date back principally to Dingle [11] whose pioneering view was able to unpin that asymptotic series solutions of the singularly perturbed differential equations related to their singular point(s) generically behave in the same standard form and they are factorially divergent. By using the Darboux's theorem [10], [12, pp. 447] which reveals why the late-order terms of asymptotic series necessarily diverge in a particular form, he described that it is the generic feature of the late terms of such series and those terms commonly occur in the factorial/power form [11, pp. 4,140]. For more detailed information on the particular type of divergence as well as the subject of exponential asymptotics, we refer the reader to see $[2,4,6,7,9]$ and references therein. The method has been also studied in [5] to discover the nature of the divergence and the leading behaviour of the later-order asymptotic expansion of integrals involving coalescing saddles. Boyd $[7,8]$ provides a list of applications where he discusses divergence for asymptotic series along with the importance of the exponentially small terms in detail. Many differential equations, including the Airy function, error function, in the form of highest derivative multiplied by a small parameter or large values of $z$ are discussed by this method, see for example $[3,14,16,18,20]$.

Email address: fatihsay@odu.edu.tr

Received: 09.12.2019; Accepted: 11.06.2020 
Exact solutions of the singular differential equations are unfortunately rare in applied mathematics and physics. Nevertheless, generating exact late-order coefficients by a recurrence relation explicitly can be very challenging or even intractable at times in general. Fortunately, it is often not essential to derive exact late-order terms, but one must make sure that they asymptotically behave in the expected specific factorial/power form [10]. In these cases, asymptotical interpretation of the differential equations plays an important role in understanding the behaviour of such differential equations, and several times accurate numerical approximations of them are enough for practical purposes. Hence, in the theory of asymptotic expansions, one is usually interested in the behaviour of the late coefficients appearing in the asymptotic expansion when their summation index $n$ is large. The late-order terms are tail of a series expansion which includes some crucial information about the divergent series when the limit $n \rightarrow \infty$. Having known the form of the late terms enables us to find, for example, optimal truncation points, exponentially small terms and potential Stokes rays. Consequently, efficient and accurate approximations for ODEs are essential. For these reasons, in this paper, in order to find the general asymptotic representation of the late-order terms of the model second order inhomogeneous differential equations, we will make use of the well-known factorial/power approach. In doing so, we represent the leading large-order approximate solution of the particular type of ODE in terms of its pre-factors. We obtain simple explicit formulas for the coefficients of the regular asymptotic expansion. The expansion coefficients are indeed simple polynomials of the pre-factors, and thus the implementations of the formulas are straightforward. We illustrate these formulas for a simple example providing all the basic information required for their practical implementations.

The main aim of this present paper is to establish formulae at a leading-order approximation for the solution of singular ODEs in terms of their pre-factors as we are only interested in discussing its asymptotic behaviour with the limits $n \rightarrow \infty$ and $\epsilon \rightarrow 0$. Upon obtaining these formulas, one can straightforwardly derive several properties of the asymptotic expansions in these limits. The outline of the paper is structured as follows: We first begin with a presentation of a model ODE whose derivatives are multiplied by a small parameter $\epsilon$ in $\S 2$. We then continue with the power series expansion of the solution of the particular ODE in powers of $\epsilon$ in the sense of Poincaré's expansion $[13,17]$ by which we obtain its leading order term and related recurrence relationship of the terms. With this in mind, we investigate its generic leading order behaviour by the factorial/power approach in §3. There we provide a general connection formula between the pre-factors of an ODE and the coefficients of its asymptotic expansion, which, in this case, permits us to have the asymptotic expansion without requiring detailed repeated dominant balance procedure. We will show that the first few terms of a complete asymptotic expansion of the ODE under consideration can be obtained by the asymptotic formulas mentioned above in terms of its pre-factors. We provide an example by which we can illustrate the implementation of the formulas in $\S 4$. Finally, we finish the paper with a brief conclusion in $\S 5$.

\section{Complete asymptotic power series of ODE}

In this paper, we consider an asymptotic expansion of the following singular second order linear inhomogeneous ODE

$$
\epsilon \frac{d^{2} w(z)}{d z^{2}}+\epsilon f(z) \frac{d w(z)}{d z}+g(z) w(z)=t(z),
$$

where the perturbation parameter $\epsilon$ is $0<\epsilon \ll 1$. The asymptotic solution of the homogeneous part of (2.1), where the highest derivative did not multiply by a small parameter, can be found, for example, in $[15,16]$. The determination of an asymptotic expansion for $w(z)$ is necessary for approximating and gathering as much information as possible for this 
type of equations with small values of $\epsilon$. Solving this type of equations sometimes would be extremely tedious. However, our aim is to know the general form of its asymptotic expansion, so does its asymptotic behaviour, not to know the exact expression of $w(z)$. To begin, assume that it has a usual Poincaré-type asymptotic expansion of the form in powers of $\epsilon$ as

$$
w(z) \sim \sum_{n=0}^{\infty} \epsilon^{n} w_{n}(z) \quad \text { as } \epsilon \rightarrow 0 .
$$

We will approximate this sum and will focus only on the terms that describe the asymptotic behaviour of the late coefficients $w_{n}(z)$ of $(2.2)$ with the limits $n \rightarrow \infty$ and $\epsilon \rightarrow 0$. The sign $\sim$ indicates that the ratio of the left and the right hand sides in (2.2) approaches 1 as $n$ increases. As it satisfies the differential equation (2.1), back-substituting the naïve asymptotic expansion (2.2) into it yields

$$
\sum_{n=0}^{\infty} \epsilon^{n+1}\left[w_{n}^{\prime \prime}(z)+f(z) w_{n}^{\prime}(z)+g(z) w_{n+1}(z)\right]+g(z) w_{0}(z)=t(z),
$$

where the prime' represents the derivative with respect to $z$. Matching both sides of the asymptotic equality (2.3) with respect to orders of $\epsilon$ gives the leading order solution at $\mathrm{O}(1)$ as

$$
w_{0}(z)=\frac{t(z)}{g(z)},
$$

wherein the zero(s) of $g(z)$ and the singularity (or singularities) of $t(z)$ are the singularities of the leading order term. Likewise, we subsequently find out the recurrence relationship of the consecutive terms of the expansion at $\mathrm{O}\left(\epsilon^{n+1}\right)$ as

$$
w_{n}^{\prime \prime}(z)+f(z) w_{n}^{\prime}(z)+g(z) w_{n+1}(z)=0 .
$$

One can uniquely define each further term of the expansion as a function of the preceding ones by using this recurrence relation. It is clear that the coefficient $w_{n+1}(z)$ is provided by the relation. We close this section by commenting on the implementation of this relation. Particularly, upon substitution of $w_{0}(z)$ given in (2.4) into (2.5), one may establish the consecutive term $w_{1}(z)$ at $\mathrm{O}(\epsilon)$. Once it is done, he sees that the power of its denominator $g(z)$ increases to 3 and notices that no new singularity is introduced by the linearity of the relation. Carrying out this differentiating process repeatedly between the consecutive terms at each order, this can be seen more clearly since all the subsequent order terms must depend on early ordered term (2.4). Therefore, the singular point of the power series expansion at every single order must be the same singularity of the leading order one, and it indeed characterizes the coefficients of the asymptotic power series in (2.2). In each succeeding order we calculate, the strength of the singular term(s) increases from the preceding order. Thus it assures the ansatz $w_{n}(z)$ to diverge infinity in the limit as $n \rightarrow \infty$. It is the actual reason for the typical factorial/power divergence of the expansion $[11]$.

\section{Application of factorial over a power form}

Because we are primarily interested in the asymptotic expansion with the limit $n \rightarrow \infty$ and because late-order terms of the expansion, $w_{n}(z)$, commonly behave like a facto$\mathrm{rial} /$ power nature in the singular perturbation, it is reasonable to assume that the lateorder terms are in this nature as

$$
w_{n}(z)=\frac{\Gamma(\alpha n+\beta)}{\chi(z)^{\alpha n+\beta}}\left(\sum_{k=0}^{\infty} \frac{A_{k}(z)}{(\alpha n)^{k}}\right) \quad \text { as } n \rightarrow \infty,
$$


where the gamma function $\Gamma$, which is one of the most widely used special function, is described in [1]. $\chi(z), \beta$ and $A_{k}(z)$ s are independent of $n$. The denominator function $\chi(z)$ is subject to the leading order singular point(s) that causes $w_{n}(z)$ to diverge, and it will later be derived by matching expansion at leading order. This function is named as the singulant by Dingle [11, pp. 6-8]. An arbitrary constant $\beta=\mathrm{O}(1)$ is added to the power of $\chi(z)$ so as to have an easy cancellation between the numerator and denominator functions when the derivatives of (3.1) are taken. Likewise, $\alpha$ is to be determined when we do the asymptotic matching at leading order in the recurrence relation. Moreover, the denominator functions appear in the powers of the subdominant exponential terms which change abruptly as Stokes rays are crossed in the complex plane. This indispensable changing behaviour of the terms is made clearer by exponential asymptotics. Substituting facto$\mathrm{rial} /$ power ansatz (3.1) into the recurrence relation after taking the associated derivatives, we find that

$$
\begin{aligned}
& \frac{(\alpha n+\beta+1)(\alpha n+\beta)\left(\chi^{\prime}(z)\right)^{2} \Gamma(\alpha n+\beta)}{\chi(z)^{\alpha n+\beta+2}} \sum_{k=0}^{\infty} \frac{A_{k}(z)}{(\alpha n)^{k}}-\frac{(\alpha n+\beta) \chi^{\prime \prime}(z) \Gamma(\alpha n+\beta)}{\chi(z)^{\alpha n+\beta+1}} \sum_{k=0}^{\infty} \frac{A_{k}(z)}{(\alpha n)^{k}} \\
& -2 \frac{(\alpha n+\beta) \chi^{\prime}(z) \Gamma(\alpha n+\beta)}{\chi(z)^{\alpha n+\beta+1}}\left(\sum_{k=0}^{\infty} \frac{A_{k}^{\prime}(z)}{(\alpha n)^{k}}\right)+\frac{\Gamma(\alpha n+\beta)}{\chi(z)^{\alpha n+\beta}}\left(\sum_{k=0}^{\infty} \frac{A_{k}^{\prime \prime}(z)}{(\alpha n)^{k}}\right) \\
& +f(z)\left[-\frac{(\alpha n+\beta) \chi^{\prime}(z) \Gamma(\alpha n+\beta)}{\chi(z)^{\alpha n+\beta+1}}\left(\sum_{k=0}^{\infty} \frac{A_{k}(z)}{(\alpha n)^{k}}\right)+\frac{\Gamma(\alpha n+\beta)}{\chi(z)^{\alpha n+\beta}}\left(\sum_{k=0}^{\infty} \frac{A_{k}^{\prime}(z)}{(\alpha n)^{k}}\right)\right] \\
& +g(z) \frac{\Gamma(\alpha n+\beta+\alpha)}{\chi(z)^{\alpha n+\beta+\alpha}}\left(\sum_{k=0}^{\infty} \frac{A_{k}(z)}{(\alpha n+\alpha)^{k}}\right)=0 .
\end{aligned}
$$

For large values of $n$, one easily sees that it approximates to

$$
\begin{aligned}
& \frac{(\alpha n)^{2}\left(\chi^{\prime}(z)\right)^{2} \Gamma(\alpha n+\beta)}{\chi(z)^{\alpha n+\beta+2}}\left(\sum_{k=0}^{\infty} \frac{A_{k}(z)}{(\alpha n)^{k}}\right)-\frac{\alpha n \chi^{\prime \prime}(z) \Gamma(\alpha n)}{\chi(z)^{\alpha n+\beta+1}}\left(\sum_{k=0}^{\infty} \frac{A_{k}(z)}{(\alpha n)^{k}}\right) \\
& -2 \frac{\alpha n \chi^{\prime}(z) \Gamma(\alpha n+\beta)}{\chi(z)^{\alpha n+\beta+1}}\left(\sum_{k=0}^{\infty} \frac{A_{k}^{\prime}(z)}{(\alpha n)^{k}}\right)+\frac{\Gamma(\alpha n+2)}{\chi(z)^{\alpha n+\beta}}\left(\sum_{k=0}^{\infty} \frac{A_{k}^{\prime \prime}(z)}{(\alpha n)^{k}}\right) \\
& +f(z)\left[-\frac{\alpha n \chi^{\prime}(z) \Gamma(\alpha n+\beta)}{\chi(z)^{\alpha n+\beta+1}}\left(\sum_{k=0}^{\infty} \frac{A_{k}(z)}{(\alpha n)^{k}}\right)+\frac{\Gamma(\alpha n+\beta)}{\chi(z)^{\alpha n+\beta}}\left(\sum_{k=0}^{\infty} \frac{A_{k}^{\prime}(z)}{(\alpha n)^{k}}\right)\right] \\
& +g(z) \frac{\Gamma(\alpha n+\beta+\alpha)}{\chi(z)^{\alpha n+\beta+\alpha}}\left(\sum_{k=0}^{\infty} \frac{A_{k}(z)}{(\alpha n+\alpha)^{k}}\right)=0 .
\end{aligned}
$$

Herein, once we use the definition of the $\Gamma$ function to balance the largest (dominant) terms for large enough $n$, we find

$$
\begin{aligned}
& \frac{(\alpha n)^{2}\left(\chi^{\prime}(z)\right)^{2} \Gamma(\alpha n+\beta)}{\chi(z)^{\alpha n+\beta+2}}\left(\sum_{k=0}^{\infty} \frac{A_{k}(z)}{(\alpha n)^{k}}\right)-\frac{\alpha n \chi^{\prime \prime}(z) \Gamma(\alpha n)}{\chi(z)^{\alpha n+\beta+1}}\left(\sum_{k=0}^{\infty} \frac{A_{k}(z)}{(\alpha n)^{k}}\right) \\
& -2 \frac{\alpha n \chi^{\prime}(z) \Gamma(\alpha n+\beta)}{\chi(z)^{\alpha n+\beta+1}}\left(\sum_{k=0}^{\infty} \frac{A_{k}^{\prime}(z)}{(\alpha n)^{k}}\right)+\frac{\Gamma(\alpha n+2)}{\chi(z)^{\alpha n+\beta}}\left(\sum_{k=0}^{\infty} \frac{A_{k}^{\prime \prime}(z)}{(\alpha n)^{k}}\right) \\
& +f(z)\left[-\frac{\alpha n \chi^{\prime}(z) \Gamma(\alpha n+\beta)}{\chi(z)^{\alpha n+\beta+1}}\left(\sum_{k=0}^{\infty} \frac{A_{k}(z)}{(\alpha n)^{k}}\right)+\frac{\Gamma(\alpha n+\beta)}{\chi(z)^{\alpha n+\beta}}\left(\sum_{k=0}^{\infty} \frac{A_{k}^{\prime}(z)}{(\alpha n)^{k}}\right)\right] \\
& +g(z) \frac{(\alpha n+\beta+\alpha-1)(\alpha n+\beta+\alpha-2) \Gamma(\alpha n+\beta+\alpha-2)}{\chi(z)^{\alpha n+\beta+\alpha}}\left(\sum_{k=0}^{\infty} \frac{A_{k}(z)}{(\alpha n+\alpha)^{k}}\right)=0 .
\end{aligned}
$$

In order to proceed and obtain an asymptotic balance between the terms of the equation (3.4), we must first derive the value of $\alpha$. To be precise, the other largest $\mathrm{O}\left(n^{2}\right)$ term, 
besides the leftmost one, is the rightmost term of (3.4). This requires that we can choose $\alpha$ by balancing the leftmost and the rightmost terms of the equation. In particular, to be able to balance the largest terms for sufficiently large values of $n$ at $\mathrm{O}\left(n^{2}\right)$, these terms of (3.4) must be of the same size whence the following holds

$$
\alpha n+\beta+2=\alpha n+\beta+\alpha,
$$

which implies that $\alpha=2$. One of the typical reasons for this is that the expansion coefficients $w_{n}(z)$ and $w_{n+1}(z)$ are consecutively related to each other by the recurrence relationship given in (2.5). Therefore, while balancing the consecutive terms of the expansion, we take $\alpha=2$ in the factorial $\Gamma(\alpha n+\beta)$ and in the powers of the denominators in the factorial/power representation. Once this value of $\alpha$ in mind is substituted into the equation (3.4), the equation turns out to be

$$
\begin{gathered}
\frac{(2 n)^{2}\left(\chi^{\prime}(z)\right)^{2} \Gamma(2 n+\beta)}{\chi(z)^{2 n+\beta+2}}\left(\sum_{k=0}^{\infty} \frac{A_{k}(z)}{(2 n)^{k}}\right)-\frac{2 n \chi^{\prime \prime}(z) \Gamma(2 n)}{\chi(z)^{2 n+\beta+1}}\left(\sum_{k=0}^{\infty} \frac{A_{k}(z)}{(2 n)^{k}}\right) \\
-2 \frac{2 n \chi^{\prime}(z) \Gamma(2 n+\beta)}{\chi(z)^{2 n+\beta+1}}\left(\sum_{k=0}^{\infty} \frac{A_{k}^{\prime}(z)}{(2 n)^{k}}\right)+\frac{\Gamma(2 n+2)}{\chi(z)^{2 n+\beta}}\left(\sum_{k=0}^{\infty} \frac{A_{k}^{\prime \prime}(z)}{(2 n)^{k}}\right) \\
+f(z)\left[-\frac{2 n \chi^{\prime}(z) \Gamma(2 n+\beta)}{\chi(z)^{2 n+\beta+1}}\left(\sum_{k=0}^{\infty} \frac{A_{k}(z)}{(2 n)^{k}}\right)+\frac{\Gamma(2 n+\beta)}{\chi(z)^{2 n+\beta}}\left(\sum_{k=0}^{\infty} \frac{A_{k}^{\prime}(z)}{(2 n)^{k}}\right)\right] \\
+g(z) \frac{(2 n+\beta+1)(2 n+\beta) \Gamma(2 n+\beta)}{\chi(z)^{2 n+\beta+2}}\left(\sum_{k=0}^{\infty} \frac{A_{k}(z)}{(2 n+2)^{k}}\right)=0 .
\end{gathered}
$$

A straightforward computation of (3.6) in which we kept only the dominant terms at $\mathrm{O}\left(n^{2}\right)$ as $n \rightarrow \infty$ shows that this equation simplifies to

$$
\frac{(2 n)^{2}\left(\chi^{\prime}(z)\right)^{2} \Gamma(2 n+\beta)}{\chi(z)^{2 n+\beta+2}}\left(\sum_{k=0}^{\infty} \frac{A_{k}(z)}{(2 n)^{k}}\right)+g(z) \frac{(2 n)^{2} \Gamma(2 n+\beta)}{\chi(z)^{2 n+\beta+2}}\left(\sum_{k=0}^{\infty} \frac{A_{k}(z)}{(2 n+2)^{k}}\right)=0,
$$

where all omitted terms are to be small comparing the remaining terms as $n \rightarrow \infty$. From this, one easily verifies that

$$
\frac{(2 n)^{2} \Gamma(2 n+\beta)}{\chi(z)^{2 n+\beta+2}}\left(\sum_{k=0}^{\infty} \frac{A_{k}(z)}{(2 n)^{k}}\right)\left(\chi^{\prime}(z)^{2}+g(z)\right)=0 \quad \text { as } n \rightarrow \infty .
$$

Because $A_{k}(z) \mathrm{s}$ for all $k$ cannot be zero, the rightmost factor of (3.8) must be equal to zero. Otherwise, in each calculation of $A_{k}(z) \mathrm{s}$ for all $k$ would become zero, which is indeed the trivial solution. Therefore, we have

$$
\chi^{\prime}(z)^{2}+g(z)=0
$$

which implies

$$
\chi^{\prime}(z)= \pm \sqrt{-g(z)}
$$

Taking the integration of both sides in (3.10) with respect to $z$ gives the determination of the form of the denominator in terms of the leading pre-factor

$$
\chi(z)=\int \pm \sqrt{-g(z)} d z+c_{\chi}
$$

where $c_{\chi}$ is a constant of integration which can be obtained by the definition of $\chi(z)$. In particular, $\chi(z)$ must satisfy the singularity of the leading order term since the singularity must be present in the late-order terms by the repeated differentiation of the early terms. Thereupon, the denominator of the late-order terms is determined by the pre-factor $g(z)$, whence (3.1) indeed holds. Next, we will establish the general form of the leading $A_{0}(z)$ in terms of the pre-factors $f(z)$ and $g(z)$ for large $n$, since rest of the terms with other indices in the summation of (3.1) will be of lower order as $n \rightarrow \infty$. To accomplish this, 
we will proceed to the next subsequent order of matching, i.e., at order $n$ by using $\alpha=2$, we find

$$
\begin{gathered}
\frac{2 n\left(\chi^{\prime}(z)\right)^{2} \Gamma(2 n+\beta)}{\chi(z)^{2 n+\beta+2}} A_{1}(z)-\frac{2 n \chi^{\prime \prime}(z) \Gamma(2 n+\beta)}{\chi(z)^{2 n+\beta+1}} A_{0}(z)-2 \frac{2 n \chi^{\prime}(z) \Gamma(2 n+\beta)}{\chi(z)^{2 n+\beta+1}} A_{0}^{\prime}(z) \\
-f(z) \frac{2 n \chi^{\prime}(z) \Gamma(2 n+\beta)}{\chi(z)^{2 n+\beta+1}} A_{0}(z)+g(z) \frac{2 n \Gamma(2 n+\beta)}{\chi(z)^{2 n+\beta+2}} A_{1}(z)=0 .
\end{gathered}
$$

Once doing the separation above, we obtain

$$
\begin{aligned}
\frac{2 n \Gamma(2 n+\beta)}{\chi(z)^{2 n+\beta+2}} & A_{1}(z)\left(\left(\chi^{\prime}(z)\right)^{2}+g(z)\right) \\
& -\frac{2 n \Gamma(2 n+\beta)}{\chi(z)^{2 n+\beta+1}}\left[\chi^{\prime \prime}(z) A_{0}(z)+2 \chi^{\prime}(z) A_{0}^{\prime}(z)+f(z) \chi^{\prime}(z) A_{0}(z)\right]=0,
\end{aligned}
$$

as $n \rightarrow \infty$. The first part of (3.13) is naturally equal to zero by the definition of the leading order condition (3.9). Hence, the second part of the equation must equal to zero that is to have

$$
\chi^{\prime \prime}(z) A_{0}(z)+2 \chi^{\prime}(z) A_{0}^{\prime}(z)+f(z) \chi^{\prime}(z) A_{0}(z)=0 .
$$

Doing the required separation above leads to the following

$$
\frac{A_{0}^{\prime}(z)}{A_{0}(z)}=-\frac{\chi^{\prime \prime}(z)+f(z) \chi^{\prime}(z)}{2 \chi^{\prime}(z)} .
$$

Direct integration of this yields

$$
\ln \left(A_{0}(z)\right)=-\frac{1}{2} \ln \left(\chi^{\prime}(z)\right)-\frac{1}{2} \int f(z) d z+c,
$$

where $c$ is an integration constant. After simplification of $(3.16), A_{0}(z)$ is subsequently obtained as

$$
A_{0}(z)=\frac{\exp \left(-\int \frac{f(z) d z}{2}\right) \exp (c)}{\sqrt{\chi^{\prime}(z)}},
$$

which can also be written out in terms of pre-factor $g(z)$ by the definition of the denominator $\chi(z)$ in (3.10) as

$$
A_{0}(z)=c_{0} \frac{\exp \left(-\int \frac{f(z) d z}{2}\right)}{\sqrt[4]{-g(z)}} .
$$

Herein, without loss of generality, $c_{0}$ is set to represent all the related constants in the solution since one can always absorb the constants into it. Collecting all the partial results obtained so far, we are now in a position to formulate our main result generated from the recursion relation via factorial/power approach that

$$
w_{n}(z) \sim c_{0} \frac{\Gamma(2 n+\beta)}{\chi(z)^{2 n+\beta}}\left(\frac{\exp \left(-\int \frac{f(z) d z}{2}\right)}{\sqrt[4]{-g(z)}}\right) \quad \text { as } n \rightarrow \infty .
$$

It is obtained in terms of the pre-factor functions of the singularly perturbed ODE. The undetermined value of $\beta$, if needed, can be determined by balancing the behaviour of the exact low-ordered term(s) with the behaviour of the late-order ansatz (3.19) with respect to $n$ since they both must behave the same near such singularity. By the leading behaviour of the late coefficients $w_{n}(z)$, we readily establish that the right-hand side of the ansatz (3.1) asymptotically equals to (3.19) with the limit $n \rightarrow \infty$. We have completely derived the behaviour and the general form of the late-order terms of a general singular ODE 
with pre-factors in the case of this paper. We finally have deduced the following regular asymptotic expansion

$$
w(z) \sim c_{0} \sum_{n=0}^{\infty} \epsilon^{n} \frac{\Gamma(2 n+\beta)}{\chi(z)^{2 n+\beta}}\left(\frac{\exp \left(-\int \frac{f(z) d z}{2}\right)}{\sqrt[4]{-g(z)}}\right) \quad \text { as } n \rightarrow \infty,
$$

which summarizes the connection formulas derived so far. In fact, it is the expansion that describes the most important behaviours of such ODEs in the limits $n \rightarrow \infty$ and $\epsilon \rightarrow 0$. Of course, the region of validity of the resulting asymptotic expression (3.20) depends only on the singularity structure of the coefficient (3.19). It can be discussed via the local analysis near the singular point(s).

\section{Illustrative example}

In this section, we give an illustrative example to demonstrate the application of the formula (3.20) developed over the course of the preceding sections for a particular form of an ODE in (2.1). While doing this, we will consider the simple explicit example of [19], i.e.,

$$
\epsilon \frac{d^{2} w(z)}{d z^{2}}-\epsilon \frac{1}{z} \frac{d w(z)}{d z}-4 z^{2} w(z)=1
$$

where $0<\epsilon \ll 1$. Upon comparing pre-factors of both equations (2.1) and (4.1), one straightforwardly notices that the corresponding pre-factors are

$$
f(z)=-\frac{1}{z}, \quad g(z)=-4 z^{2} \quad \text { and } \quad t(z)=1 .
$$

First, the usual approach in the asymptotic procedure is to expand the solution $w(z)$ of (4.1) as a power series of $\epsilon$ as in (2.2). Then it is easy to see that the singularity of such expansion occurs at $z=0$ by the definition of the leading order term given in (2.4). After making use of the formulae obtained for $\chi(z)$ and $A_{0}(z)$ in (3.11) and (3.18), respectively, one can directly find out, after some computations, with the factorial divided by power ansatz (3.1) in mind that the associated components of the ansatz with the limit $n \rightarrow \infty$ are

$$
\chi(z)=z^{2} \quad \text { and } \quad A_{0}(z)=\mp \frac{1}{\sqrt{2}},
$$

where $\chi(z)$ is derived subject to the singularity of the leading term. This completes the derivation of the late-order terms in the asymptotic expansion for large $n$ and small $\epsilon$. To be precise, the substitution of these into the expansion (3.20) directly provides an explicit approximation for certain pre-factors related to the coefficients of the expansion without the need of repeated dominant balance procedure as it is already done in our analysis, and this leads to the following asymptotic expression

$$
w(z) \sim \mp c_{0} \sum_{n=0}^{\infty} \epsilon^{n} \frac{\Gamma(2 n+\beta)}{\sqrt{2} z^{4 n+2 \beta}} \quad \text { as } n \rightarrow \infty .
$$

This is almost exactly the same as the equation (45) of [19] for $\beta=1$ with an appropriate value of the constant $c_{0}$.

\section{Conclusion}

In this paper, we successfully determined the general asymptotic representation of the late-order terms in the asymptotic power series (2.2) of the model ODE (2.1) in terms of their pre-factors. In particular, we derived formulae whose computation and implementation are straightforward for the particular type of singular ODEs in the form of this paper. 
Their leading order behaviour was investigated based on the use of a factorial/power representation. Using these formulas for such equations, one can straightforwardly interpret the several properties of singular ODEs as in (2.1) mentioned in the introduction and their related asymptotic expansions (2.2) more generally. The asymptotic expression (3.20) is suitable for numerical computation for sufficiently large values of $n$ and for small values of $\epsilon$.

Acknowledgment. The author is grateful to the anonymous referees for their useful comments and suggestions on the manuscript.

\section{References}

[1] M. Abramowitz and I.A. Stegun, (editors), Handbook of Mathematical Functions with Formulas, Graphs, and Mathematical Tables, Reprint of the 1972 edition, Dover Publications, Inc., New York, 1992.

[2] K.L. Adams, Exponential asymptotics, Ph.D. Thesis, University of Nottingham, Nottingham, UK, 1997.

[3] C.M. Bender and S.A. Orszag, Advanced Mathematical Methods for Scientists and Engineers I: Asymptotic Methods and Perturbation Theory, Reprint of the 1978 original, Springer-Verlag, New York, 1999.

[4] M.V. Berry, Uniform asymptotic smoothing of Stokes's discontinuities, Proc. Roy. Soc. London Ser. A 422 (1862), 7-21, 1989.

[5] M.V. Berry and C.J. Howls, Unfolding the high orders of asymptotic expansions with coalescing saddles: singularity theory, crossover and duality, Proc. Roy. Soc. London Ser. A 443 (1917), 107126, 1993.

[6] M.V. Berry, Asymptotics, superasymptotics, hyperasymptotics..., in: Asymptotics Beyond All Orders, NATO Adv. Sci. Inst. Ser. B Phys. 284, 1-14, H. Segur, S. Tanveer, H. Levine (editors), Springer Science \& Business Media, Boston, 1991.

[7] J.P. Boyd, The devil's invention: asymptotic, superasymptotic and hyperasymptotic series, Acta Appl. Math. 56 (1), 1-98, 1999.

[8] J.P. Boyd, Hyperasymptotics and the linear boundary layer problem: why asymptotic series diverge, SIAM Rev. 47 (3), 553575, 2005.

[9] S.J. Chapman and D.B. Mortimer, Exponential asymptotics and Stokes lines in a partial differential equation, Proc. R. Soc. Lond. Ser. A Math. Phys. Eng. Sci. 461 (2060), 2385-2421, 2005.

[10] G. Darboux, Mémoire sur l'approximation des fonctions de très-grands nombres, et sur une classe étendue de développements en série, (French), J. Math. Pures Appl. 4, 556, 1878.

[11] R.B. Dingle, Asymptotic Expansions: Their Derivation and Interpretation, Academic Press, London-New York, 1973.

[12] P. Henrici, Applied and Computational Complex Analysis: Special Functions - Integral Transforms - Asymptotics - Continued Fractions, 2, Reprint of the 1977 original, John Wiley \& Sons, Inc., New York, 1991.

[13] E.J. Hinch, Perturbation Methods, Cambridge Texts in Applied Mathematics, Cambridge University Press, Cambridge, 1991.

[14] P.J. Langman, When is a Stokes line not a Stokes line?, Ph.D. Thesis, University of Southampton, Southampton, UK, 2005.

[15] F.W.J. Olver, Asymptotic expansions of the coefficients in asymptotic series solutions of linear differential equations, Methods Appl. Anal. 1 (1), 1-13, 1994.

[16] F.W.J. Olver, Asymptotics and Special Functions, Reprint of the 1974 original, A K Peters, Ltd., Wellesley, MA, 1997. 
[17] H. Poincaré, Sur les intégrales irrégulières: Des équations linéaires (French), Acta Math. 8 (1), 295-344, 1886.

[18] F. Say, Exponential asymptotics: multi-level asymptotics of model problems, Ph.D. Thesis, University of Nottingham, Nottingham, UK, 2016.

[19] S. Tanveer, Viscous displacement in a Hele-Shaw cell, in: Asymptotics Beyond All Orders, NATO Adv. Sci. Inst. Ser. B Phys. 284, 131-153, H. Segur, S. Tanveer, H. Levine (editors), Springer Science \& Business Media, Boston, MA, 1991.

[20] W. Wasow, Asymptotic Expansions for Ordinary Differential Equations, Reprint of the 1965 edition, Robert E. Krieger Publishing Co., Huntington, NY, 1976. 